\title{
Confiabilidade do Canal em Sistemas DS/CDMA com Codificação Turbo Sujeitos ao Desvanecimento Lento e Seletivo em Frequiência
}

\author{
Wagner Okano, Fernando Ciriaco, Taufik Abrão
}

\begin{abstract}
Resumo-Este trabalho analisa as estratégias de codificação turbo e convolucional aplicadas ao problema da detecção uniusuário (SuD - Single User Detection) em sistemas de múltiplo acesso DS/CDMA ( Direct Sequence-Code Division Multiple Access) considerando canais de multipercurso com desvanecimento lento e seletivo em freqüência. Propõe-se uma nova equação para o fator de confiabilidade do canal DS/CDMA, obtida a partir de resultados de desempenho de simulação Monte Carlo (MCS). A comparação com resultados da literatura indicou superioridade no desempenho na região de alta $\frac{E_{b}}{N_{0}}$ e similaridade para as demais regiões. Adicionalmente, analisa-se o compromisso complexidade/requisito de memória versus desempenho de receptores DS/CDMA considerando decodificadores turbo (padrão cdma2000) e Viterbi (padrão IS-95).
\end{abstract}

Palavras-Chave-Confiabilidade do Canal, Códigos Turbo, DS/CDMA, Detecção uniusuário, Canais de Multipercurso.

Abstract-This work analyzes the Turbo and Convolutional codes strategies applied to the single-user detection $(\mathrm{SuD})$ problem in multiple access systems DS/CDMA, in multipath slow Rayleigh channels. In this work a new equation for DS/CDMA channel reliability factor is proposed. Obtained from Monte Carlo simulations (MCS), this equation was compared with the literature results and has indicated a superior performance in the high $\frac{E_{b}}{N_{0}}$ regions; in others $\frac{E_{b}}{N_{0}}$ regions the proposed equation has similar performance than those described in the literature. Additionally, this work makes an analysis of the complexity/memory requirements versus performance of DS/CDMA receivers considering turbo (cdma2000) and Viterbi (IS-95) decoders.

Keywords-Channel Reliability, Turbo-Codes, DS/CDMA, Single-user Detection, Multipath Channels.

\section{INTRODUÇÃO}

O desvanecimento e a interferência de múltiplo acesso (MAI - Multiple Access Interference) são os dois principais fatores que limitam o desempenho em sistemas DS/CDMA. Em particular, detectores multiusuário (MuD - Multiuser Detection) são utilizados para combater a MAI, enquanto a diversidade espaço-temporal é utilizada no combate ao desvanecimento. A combinação de códigos corretores de erro no receptor (FEC Forward Error Correction) é utilizada para combater a MAI e o desvanecimento de forma conjunta.

A codificação é freqüentemente usada em sistemas de comunicação digital para proteger a informação do ruído e

Wagner Okano é Engenheiro de Telecomunicações da Sercomtel Celular S.A, Londrina, PR. Atualmente é estudante do programa de mestrado do Departamento de Engenharia Elétrica da Universidade Estadual de Londrina (DEEL-UEL); e-mail: wjokano@sercomtel.com.br.

Fernando Ciriaco e Taufik Abrão são Docentes do Departamento de Engenharia Elétrica da Universidade Estadual de Londrina - DEEL-UEL; fciriaco@uel.br, taufik@uel.br da interferência e deste modo reduzir a degradação de desempenho no receptor, com a inserção de bits de redundância no sinal de informação. O uso da codificação implica em uma redução na taxa de transmissão ou uma expansão na largura da banda.

Desde 1993, inúmeras pesquisas têm sido realizadas na área de codificação turbo [1]; tais investigações têm por objetivo reduzir a complexidade associada ao decodificador. Constituem decodificadores práticos de complexidade factível os algoritmos Max-Log-MAP sugerido por Koch e Baier [2] e também por Erfanian et.al. [3], o algoritmo Log-MAP sugerido por Robertson et.al. [4] e o SOVA sugerido por Hagenauer e Hoeher [5], [6].

Neste trabalho serão utilizadas as duas principais estratégias de decodificação existentes: a de máxima probabilidade a posteriori (MAP - Maximum A Posteriori Probability) [7] e o algoritmo de Viterbi (VA - Viterbi Algorithm) [8], [9].

O presente trabalho está dividido em VI seções. A seção II descreve o modelo do sistema DS/CDMA em canais de multipercurso com desvanecimento e também o princípio da codificação convolucional da informação. A seção III revisa as estratégias de decodificação turbo e o algoritmo de Viterbi, bem como propõe uma equação para a medida da confiabilidade de canal. Os resultados numéricos de desempenho para a codificação turbo e para o algoritmo de Viterbi, em termos de BER, são apresentados na seção IV. A seção V analisa a complexidade computacional dos codificadores e decodificadores em termos do número de transições na treliça e do requisito de memória. Por fim, a seção VI sintetiza as principais conclusões deste trabalho.

\section{Modelo De Sistema DS/CDMA}

Em um sistema DS/CDMA com modulação por chaveamento de fase binária (BPSK - Binary Phase-Shift Keying) o sinal transmitido pelo $k$-ésimo usuário em um sistema com $K$ usuários assíncronos ativos é dado por [10], [11]:

$$
z_{k}(t)=\sqrt{2 P_{k}} \sum_{i} x_{k}^{(i)} s_{k}\left(t-i T_{b}-\tau_{k, \ell}\right) \cos \left(\omega_{c} t\right)
$$

onde $P_{k}=A_{k}^{2} / 2$ representa a potência do $k$-ésimo usuário; $x_{k}^{(i)}$ é o $i$-ésimo símbolo BPSK codificado com período $T_{b}$, relacionado ao $k$-ésimo bit de informação na codificação turbo, $\omega_{c}$ é a freqüência angular da portadora; $s_{k}(t)=$ $\sum_{n=0}^{N-1} p\left(t-n T_{c}\right) \underline{s}_{k, n}$ é a sequência de espalhamento no intervalo $\left[0, T_{b}\right)$ e zero fora, onde $\underline{s}_{k, n} \in\{-1 ; 1\}$ é o $n$-ésimo 
chip da seqüência de comprimento $N$ utilizada pelo $k$-ésimo usuário; $T_{c}$ é o período de chip e o ganho de processamento $N=\frac{T_{b}}{T_{c}}$; assume-se formatação de pulso $p(t)$ retangular com amplitude $\frac{1}{\sqrt{N}}$ no intervalo $\left[0 ; T_{c}\right)$ e zero fora; $\tau_{k, \ell}=\Delta_{k, \ell}+d_{k}$ representa um atraso aleatório no $[0 ; N-1] T_{c}$, onde $d_{k}$ constitui o atraso de propagação e $\Delta_{k, \ell} \mathrm{o}$ atraso do $\ell$-ésimo percurso para o $k$-ésimo usuário.

Assumindo um pacote de dados (frame) com $I$ bits para cada usuário, propagando-se através de $L$ percursos independentes com desvanecimento Rayleigh lento, o sinal recebido em banda base (assumindo-se filtro passa-baixa ideal) e notação vetorial é dado por [11]:

$$
r(t)=\sum_{i=0}^{I-1} \mathbf{s}^{T}\left(t-i T_{b}\right) \mathbf{a c}^{(i)} \mathbf{b}^{(i)}+\eta(t)
$$

onde: $\mathbf{s}(t)=\left[s_{1}\left(t-\tau_{1,1}\right), s_{1}\left(t-\tau_{1,2}\right), \ldots, s_{1}(t-\right.$ $\left.\left.\tau_{1, L}\right) ; \ldots ; s_{k}\left(t-\tau_{k, \ell}\right), \ldots, s_{K}\left(t-\tau_{K, L}\right)\right]^{T}$ é a matriz das seqüências de espalhamento, a = $\operatorname{diag}\left[\sqrt{P_{1}^{\prime}} \mathbf{I}, \sqrt{P_{2}^{\prime}} \mathbf{I}, \ldots, \sqrt{P_{K}^{\prime}} \mathbf{I}\right]$ é a matriz diagonal das amplitudes recebidas incluindo os efeitos de perda de percurso e sombreamento, onde $\mathbf{I}_{L \times L}$ é a matriz identidade de dimensão $L ; \mathbf{c}^{(i)}=\operatorname{diag}\left[c_{1,1}^{(i)}, \ldots, c_{1, L}^{(i)}, c_{2,1}^{(i)}, \ldots, c_{2, L}^{(i)}, \ldots, c_{K, L}^{(i)}\right]$ é a matriz diagonal de ganho de canal, e o vetor de dados é dado por $\mathbf{b}^{(i)}=\left[\mathbf{b}_{1}^{(i)}, \mathbf{b}_{2}^{(i)}, \ldots, \mathbf{b}_{K}^{(i)}\right]^{T}$ com $\mathbf{b}_{k}^{(i)}$ representando o vetor de bits com dimensão $1 \times L$ referente ao $k$-ésimo usuário.

A saída do filtro casado considerando uma recepção coerente para o $k$-ésimo usuário correspondente ao $\ell$-ésimo componente multipercurso amostrado ao final do $i$-ésimo intervalo de bit é $\int_{-\infty}^{+\infty} r(t) s_{k}\left(t-i T_{b}-\tau_{k, \ell}\right) d t$, resultando em:

$$
u_{k, \ell}^{(i)}=\sqrt{P_{k}^{\prime}} T_{b} \Psi_{k, \ell}^{(i)} b_{k}^{(i)}+S I_{k, \ell}^{(i)}+I_{k, \ell}^{(i)}+n_{k, \ell}^{(i)}
$$

onde o primeiro termo corresponde ao sinal desejado, o segundo à auto-interferência, o terceiro à interferência de múltiplo acesso sobre o $\ell$-ésimo componente multipercurso do $k$-ésimo usuário e o último ao AWGN (Additive White Gaussian Noise) filtrado.

\section{DeCODificAÇAO E CONFIABILIDAdE do CANAL}

A seguir, analisa-se o desempenho do critério MAP e do algoritmo de Viterbi, considerando suas respectivas complexidades, bem como propõe-se uma nova equação para a medida da confiabilidade de canal.

\section{A. Algoritmo BCJR}

Bahl et.al. [7] publicaram um algoritmo de decodificação baseado em probabilidades a posteriori denominado algoritmo BCJR, algoritmo MAP ou ainda "forward-backward algorithm”. Entretanto, uma vez que o BCJR possui uma maior complexidade que o algoritmo de Viterbi, durante cerca de duas décadas tal decodificador não foi utilizado na prática, até o advento dos códigos turbo em 1993 [1].

Existem diversas versões simplificadas do algoritmo MAP, denominadas log-MAP (Logarithmic Maximum a Posteriori
Probability) [4] e max-log-MAP (Maximum Logarithmic Maximum a Posteriori Probability) [12], [13].

$\mathrm{O}$ algoritmo BCJR, ou qualquer outro, procurará estimar a seqüência de bits originais $b_{k}^{(i)}$ a partir da seqüência codificada $\mathbf{y}_{k}$. Neste contexto, a razão de verossimilhança logarítmica (LLR) a posteriori é dada por [7]:

$$
L\left(b_{k}^{(i)} \mid \mathbf{y}_{k}\right)=\ln \frac{\sum_{R_{1}} P\left(s^{\prime}, s, \mathbf{y}_{k}\right)}{\sum_{R_{0}} P\left(s^{\prime}, s, \mathbf{y}_{k}\right)}=\ln \frac{\sum_{R_{1}} \alpha_{i-1}\left(s^{\prime}\right) \gamma_{i}\left(s^{\prime}, s\right) \beta_{i}(s)}{\sum_{R_{0}} \alpha_{i-1}\left(s^{\prime}\right) \gamma_{i}\left(s^{\prime}, s\right) \beta_{i}(s)}
$$

onde $P\left(s^{\prime}, s, \mathbf{y}_{k}\right)$ representa a probabilidade conjunta de estar no estado $s$ ' no instante $i-1$, estar no estado $s$ no instante corrente $i$, e de a sequiência de $I$ bits recebida ser $\mathbf{y}_{k}$. No numerador, $R_{1}$ significa que o somatório se estende às transições entre estados $s^{\prime}$ e $s$ provocadas por um bit $b_{k}^{(i)}=+1$. Da mesma maneira, no denominador, $R_{0}$ designa os outros ramos, originados por um bit $b_{k}^{(i)}=-1$.

A probabilidade conjunta $P\left(s^{\prime}, s, \mathbf{y}_{k}\right)$ pode ser obtida pelo produto de outras três probabilidades, da forma:

$$
P\left(s^{\prime}, s, \mathbf{y}_{k}\right)=\alpha_{i-1}\left(s^{\prime}\right) \gamma_{i}\left(s^{\prime}, s\right) \beta_{i}(s)
$$

No instante $i$, as probabilidades $\alpha, \gamma$ e $\beta$ estão associadas ao passado, presente e futuro da seqüência $\mathbf{y}_{k}$, respectivamente. A probabilidade $\gamma_{i}\left(s^{\prime}, s\right)$ é a probabilidade condicional de receber o símbolo $\mathbf{y}_{k}^{(i)}$ no instante $i$ e o estado atual ser $S_{i}=s$, dado que o estado anterior foi $S_{i-1}=s^{\prime}$. Para canais AWGN, essa probabilidade é composta pelo produto de probabilidades:

$$
\begin{aligned}
& y_{k}^{(i)}\left(s^{\prime}, s\right)=P\left(y_{k}^{(i)} \mid x_{k}^{(i)}\right) P\left(b_{k}^{(i)}\right) \\
& =C_{i} \exp \left(b_{k}^{(i)} L\left(b_{k}^{(i)}\right) / 2\right) \exp \left(\frac{L_{c}}{2} \sum_{l=1}^{n} x_{k, l}^{(i)} y_{k, l}^{(i)}\right)
\end{aligned}
$$

onde $C_{i}$ é irrelevante no cálculo da confiabilidade da estimativa, pois aparece no numerador e no denominador da equação (4), sendo cancelado ao se calcular a LLR condicional $L\left(b_{k}(i) \mid \mathbf{y}_{k}\right)$. Os termos $x_{k, l}^{(i)}$ e $y_{k, l}^{(i)}$ representam o bit $l$ no instante $i$ da seqüência à saída do codificador e à entrada do decodificador, respectivamente. $\mathrm{O}$ termo $L_{c}$ é denominado valor ou medida de confiabilidade do canal, sendo descrita na literatura como [14], [15]:

$$
L_{c}=4 \Psi \frac{E_{c}}{N_{0}}=4 \Psi R_{c} \frac{E_{b}}{N_{0}}
$$

onde $E_{c}$ e $E_{b}$ são as energias transmitidas por bit codificado e bit de informação, respectivamente, $R_{c}$ é a taxa do código e $\Psi$ é a amplitude de desvanecimento. No caso de canal puramente AWGN, $\Psi=1$.

\section{B. Medida da Confiabilidade do Canal}

Resultados de desempenho via método MCS mostraram que a aplicação da equação (7) resulta em bons desempenhos para a estratégia turbo apenas nas regiões de baixo e médio $\frac{E_{b}}{N_{0}}$. Para valores de $\frac{E_{b}}{N_{0}}>14 \mathrm{~dB}$, o desempenho é degradado consideravelmente, tornando sua utilização inviável. Baseado na técnica de ajuste de curvas (fitting) do tipo sigmóide, tendo como base desempenhos MCS, obteve-se, de forma 
não-exaustiva, a seguinte expressão para a medida da confiabilidade do canal com desvanecimentos Rayleigh lentos e seletivos em frequiência:

$$
L_{c}=\frac{-16,6785}{1+\exp \left(\frac{\frac{E_{b}}{N_{0}}(d B)-8,8324}{2,7642}\right)}+17,622
$$

Tal expressão permite a obtenção de desempenhos apreciáveis para o sistema com codificação turbo, tanto nas faixas de baixo e médio $\frac{E_{b}}{N_{0}}$ (similares aos reportados na literatura) quanto na região de alto $\frac{E_{b}}{N_{0}}$. No caso da região de alta $\frac{E_{b}}{N_{0}}$ ), desempenhos MCS mostraram-se superiores aos relatados na literatura. Um resultado ilustrativo de desempenho, obtido via simulação MCS, comparando a eficácia da utilização da medida de confiabilidade do canal, expressa pelas equações (7) versus (8), é apresentado na seção IV, figura 3.

Ressalte-se que exitem poucos estudos na literatura considerando a equação da confiabilidade do canal para alta região de $\frac{E_{b}}{N_{0}}$. Neste trabalho, considerou-se a estratégia indireta de obtenção de uma expressão fechada para a confiabilidade do canal a partir dos resultados de desempenho do sistema em termos de taxa de erro de bit. A equação (8) constitui a contribuição deste trabalho.

\section{Decodificação Turbo}

A aplicação do algoritmo BCJR à decodificação iterativa resulta na estratégia conhecida como decodificação Turbo. Considerando uma seqüência codificada com taxa $1 / n$ no qual o primeiro bit codificado, $x_{k, 1}^{(i)}$, é igual ao bit de informação $b_{k}^{(i)}$, a LLR a posteriori pode ser decomposta numa soma de três parcelas [1]:

$$
L\left(b_{k}^{(i)} \mid \mathbf{y}_{k}\right)=L\left(b_{k}^{(i)}\right)+L_{c} y_{k, 1}^{(i)}+L_{e}\left(b_{k}^{(i)}\right)
$$

As primeiras duas parcelas estão relacionadas com o bit de informação $x_{k}^{(i)}$. No entanto, a terceira, $L_{e}\left(b_{k}^{(i)}\right)$, depende somente dos bits de paridade da palavra de código, denominada de informação extrínseca. Por subtração, a estimativa de $L\left(b_{k}^{(i)}\right)$ pode ser obtida:

$$
L_{e}\left(b_{k}^{(i)}\right)=L\left(b_{k}^{(i)} \mid \mathbf{y}_{k}\right)-L\left(b_{k}^{(i)}\right)-L_{c} y_{k, 1}^{(i)}
$$

Presume-se que esta estimativa de $L\left(b_{k}^{(i)}\right)$ seja um valor mais preciso da LLR, devendo, a priori substituir o valor anterior de $L\left(b_{k}^{(i)}\right)$. Caso esse procedimento seja repetido de um modo iterativo, fornecendo a um outro decodificador os valores de $L_{c} y_{k, 1}^{(i)}$ e a nova $L\left(b_{k}^{(i)}\right)=L_{e}\left(b_{k}^{(i)}\right)$, espera-se obter uma $L\left(b_{k}^{(i)} \mid \mathbf{y}_{k}\right)$ mais confiável a cada nova iteração. A utilização dessa estratégia diversas vezes (utilizando a equação (9)) caracteriza a decodificação Turbo.

Os inventores dos códigos turbo [1] usaram dois códigos convolucionais recursivos e sistemáticos (RSC), de taxa 1/2, concatenados em paralelo e entrelaçados, e realizaram a decodificação iterativamente com dois decodificadores MAP, figuras 1 e 2 , onde $\mathrm{P}$ e $\mathrm{P}^{-1}$ representam o entrelaçador e o desentrelaçador, respectivamente.

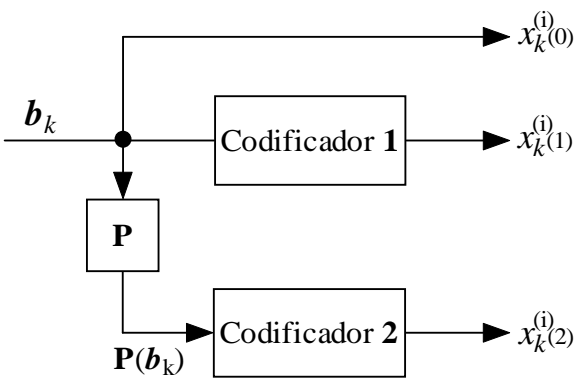

Fig. 1. Codificador Turbo.

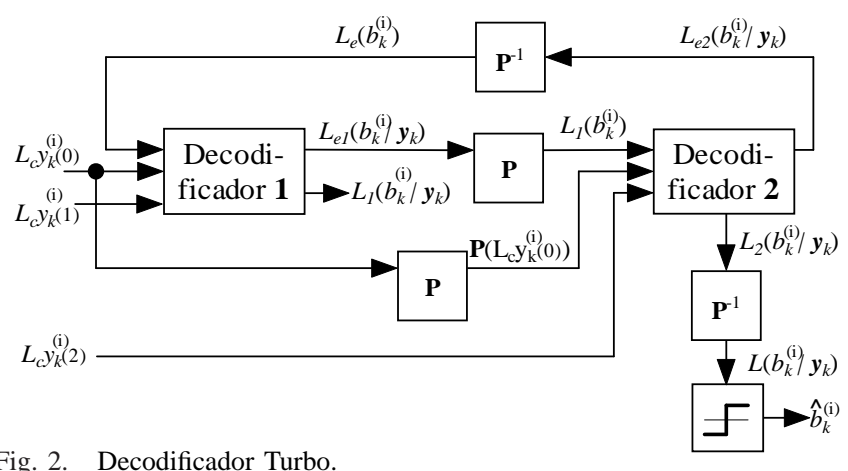

Fig. 2. Decodificador Turbo.

\section{Resultados Numéricos}

Em todas as simulações Monte Carlo, os seguinte parâmetros de sistema foram adotados: número mínimo de erros/ponto $=10$; seqüências de espalhamento aleatórias com ganho de processamento $N=31$; canal com dois percursos e desvanecimento lento seguindo a distribuição Rayleigh com atrasos aleatórios ordenados e uniformente distribuídos no intervalo $[0 ; N-1] T_{c}$ e perfil atraso-potência com $\mathbb{E}\left[\Psi_{k, 1}^{2}\right]=$ 0,652 e $\mathbb{E}\left[\Psi_{k, 2}^{2}\right]=0,348, \forall k$ e carregamento variável com o número de usuários ativos $K=[1,5,10,20]$, dependendo do sistema analisado, resultando em um carregamento, $L_{N}=\frac{K}{N}$.

Para efeito de comparação foram incluídos o desempenho Monte Carlo para o detector convencional (Rake) e o limite analítico quando não há codificação e nem MAI no sistema (SuB - Single User Bound) [10]:

$$
B E R_{\mathrm{SuB}}=\frac{1}{2} \sum_{\ell=1}^{D}\left\{\left[1-\sqrt{\frac{\bar{\gamma}_{\ell}}{\bar{\gamma}_{\ell}+1}}\right] \prod_{i, i \neq \ell}^{D} \frac{\bar{\gamma}_{\ell}}{\bar{\gamma}_{\ell}+\bar{\gamma}_{i}}\right\}
$$

onde $D$ é a diversidade Rake (número de ramos no receptor); neste trabalho, adotou-se $D=L$ e $\bar{\gamma}_{\ell}$ o valor médio quadrático do $\ell$-ésimo sinal recebido. Utilizou-se a relação sinal-ruído média no receptor (SNR - signal-to-noise ratio), $\bar{\gamma}=\sum_{\ell=1}^{D} \bar{\gamma}_{\ell}$, no intervalo $\bar{\gamma}=[0 ; 16] \mathrm{dB}$ para decodificação Turbo e $\bar{\gamma}=$ $[0 ; 14] \mathrm{dB}$ para decodificador de Viterbi.

A cada realizaçao MCS, assume-se que todos os $K$ usuários apresentem velocidades constantes e uniformemente distribuídas no intervalo $\left[0 ; v_{\max }\right]$, resultando em uma frequência Doppler máxima de $f_{D}=\frac{v_{\max }}{\lambda_{c}}=222,2 \mathrm{~Hz}$ para uma frequência da portadora $f_{c}=\frac{1}{\lambda_{c}}=2 G H z$.

A Tabela I sintetiza os principais parâmetros do sistema adotados nas simulações MCS. 
TABELA I

PRINCIPAIS PARÂMETROS DO SISTEMA.

\begin{tabular}{|c|c|c|c|c|}
\cline { 3 - 4 } \multicolumn{2}{c|}{} & \multicolumn{2}{c|}{$\bar{\gamma}$} \\
\hline Sistema & $K$ & $L_{N}(\%)$ & Convolucional & Turbo \\
\hline S1 & 1 & $3,23 \%$ & {$[0,14] \mathrm{dB}$} & {$[0,16] \mathrm{dB}$} \\
\hline S2 & 5 & $16,13 \%$ & {$[0,14] \mathrm{dB}$} & {$[0,16] \mathrm{dB}$} \\
\hline S3 & 10 & $32,26 \%$ & {$[0,14] \mathrm{dB}$} & {$[0,16] \mathrm{dB}$} \\
\hline S4 & 20 & $64,52 \%$ & {$[0,14] \mathrm{dB}$} & {$[0,16] \mathrm{dB}$} \\
\hline
\end{tabular}

Adicionalmente, adotou-se a matriz geradora dos códigos Turbo da forma [1, 15/13, 17/13] em octal. Para a codificação convolucional adotou-se os polinômios geradores, dado em octal, conforme indicado na tabela II.

TABELA II

Polinômios DA CODIFICAÇÃo CONVOLUCIONAL.

\begin{tabular}{|c|c|c|c|c|c|}
\hline Taxa & Número de & \multicolumn{4}{|c|}{ Polinômio } \\
\cline { 3 - 6 } $1 / n$ & Registradores $Q$ & $g_{0}$ & $g_{1}$ & $g_{2}$ & $g_{3}$ \\
\hline \hline $1 / 2$ & 8 & 753 & 561 & - & - \\
\hline $1 / 3$ & 8 & 557 & 663 & 711 & - \\
\hline $1 / 4$ & 8 & 765 & 671 & 513 & 473 \\
\hline
\end{tabular}

O desempenho dos algoritmos de Viterbi e Turbo foram analisadas para as três taxas de codificação, 1/2, 1/3 e 1/4, acarretando numa taxa básica de transmissão e tamanho dos pacotes que podem ser observados na tabela III.

TABELA III

PARÂMETRos dos ALGORITMOS DE Viterbi E TURBo.

\begin{tabular}{|c|c|}
\hline Cenário & $1 / n$ \\
\hline 1 & $1 / 2$ \\
\hline 2 & $1 / 3$ \\
\hline 3 & $1 / 4$ \\
\hline
\end{tabular}

\begin{tabular}{|c|c|c|c|}
\hline \multicolumn{2}{|c|}{ Viterbi } & \multicolumn{2}{c|}{ Turbo } \\
\hline$I$ & $R_{b}(\mathrm{bps})$ & $I$ & $R_{b}(\mathrm{bps})$ \\
\hline 280 & 14400 & 282 & 14400 \\
\hline 184 & 9600 & 186 & 9600 \\
\hline 128 & 6800 & 130 & 6800 \\
\hline
\end{tabular}

Uma comparação de desempenho empregando-se as equações (7) e (8) é apresentada na figura 3. Para as faixas de baixo e médio $\bar{\gamma}$, o emprego da equação (8) resulta em desempenho similar ao reportando na literatura, equação (7); adicionalmente, resulta em desempenho consistente para a faixa de alto $\bar{\gamma}$. Verifique-se que o emprego da medida de confiabilidade de canal reportado na literatura torna-se inconsistente para alta região de SNR. Neste sentido, a equação proposta torna-se uma melhor opção para a determinação da confiabilidade do canal na região de alta SNR.

A figura 4 apresenta o desempenho do decodificador Turbo com 5 iterações considerando diferentes índices de carregamento (sistemas S1, S2, S3 e S4) e cenário 2. Note-se que o desempenho da estratégia Turbo mostra-se melhor que o SuB, considerando modulação BPSK e ausência de codificação, para toda a faixa de $\bar{\gamma}$ e número de usuários simulado, mostrando ser uma estratégia capaz de garantir uma baixa BER mesmo para pequenos valores de $\bar{\gamma}$ quando em canal multipercurso. Observa-se que para garantir uma BER $<10^{-4}$ e considerando $K<10$ usuários, é necessário apenas uma relação de $\bar{\gamma}<$ $16 \mathrm{~dB}$. No entanto, quando aumenta-se o carregamento o desempenho é degradado proporcionalmente, mostrando que a estratégia Turbo é sensível ao aumento do número de usuários no sistema. Quando o carregamento aumentar deve-se utilizar

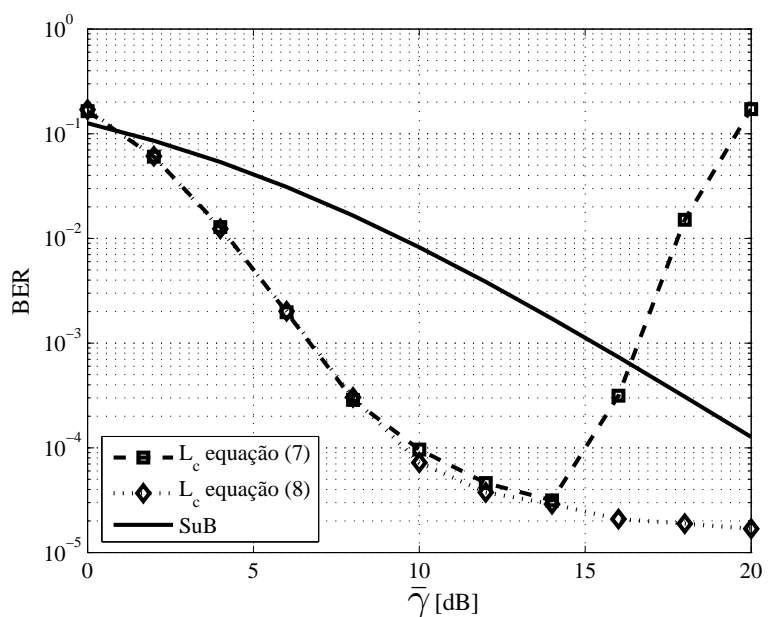

Fig. 3. Comparação das equações (7) e (8) para sistema S2 e cenário 2 e SuB, equação (11).

de forma combinada uma maior taxa de codificação, uma melhor estratégia para o entrelaçador e desentrelaçador e/ou um maior número de iterações visando atingir a BER desejada.

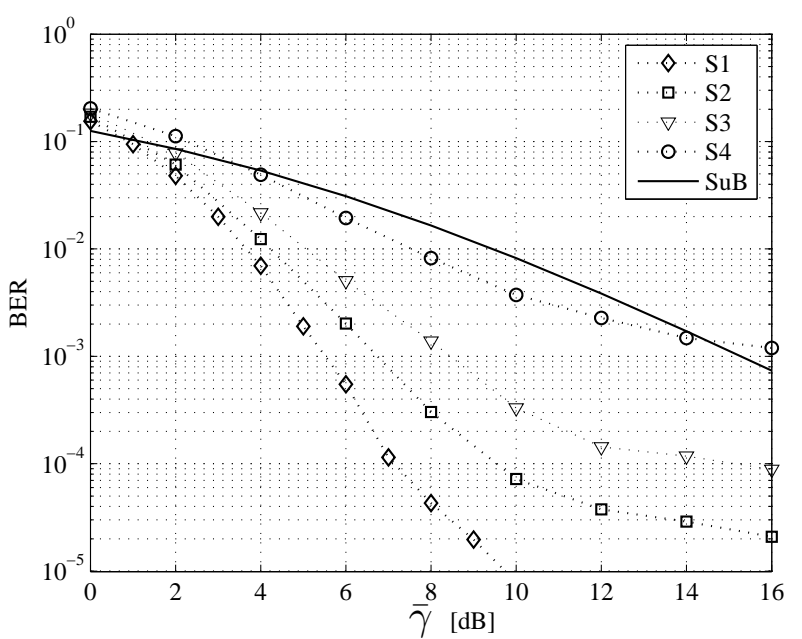

Fig. 4. Desempenho para decodificação Turbo em cenário 2 e $N_{\text {iter }}=5$.

A figura 5 apresenta o desempenho do decodificador de Viterbi considerando diferentes índices de carregamento (sistemas S1, S2, S3 e S4) e cenário 2. Note-se que o desempenho do decodificador de Viterbi também é melhor que o SuB, considerando modulação BPSK e ausência de codificação, para toda a faixa de $\bar{\gamma}$ e número de usuários simulado, garantindo também uma baixa BER mesmo para pequenos valores de $\bar{\gamma}$ quando em canal multipercurso. No entanto, quando o carregamento do sistema é incrementado, o desempenho é degradado proporcionalmente, mostrando-se mais sensível ao aumento de carregamento do que o decodificador Turbo, quando na condição do cenário 2 (taxa $=1 / n=1 / 3)$.

A figura 6 apresenta o desempenho dos decodificadores de Viterbi e Turbo considerando diferentes taxas de codificação (cenários 1, 2 e 3) e carregamento do sistema S3 ( $K=10$ usuários). Note-se o enorme ganho de desempenho de ambas as técnicas de codificação em relação ao detector Rake para toda a faixa de $\bar{\gamma}$ simulada com exceção da codificação 


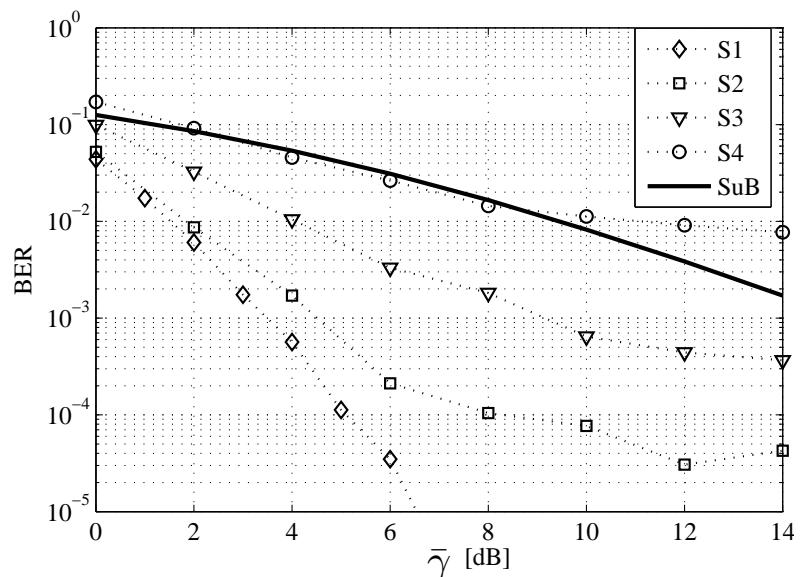

Fig. 5. Desempenho para o decodificador de Viterbi em cenário 2.

convolucional de taxa $1 / 2$ e relação $\bar{\gamma}<4 \mathrm{~dB}$. Esse ganho de desempenho mostra-se mais acentuado à medida que a relação $\bar{\gamma}$ aumenta. Quando se compara o decodificador Turbo com o de Viterbi observa-se que para taxa de codificação pequena, $1 / 2$, o decodificador Turbo apresenta melhor desempenho para toda a faixa de $\bar{\gamma}$ simulada e para taxa de codificação de $1 / 3$ para a faixa de $\bar{\gamma}>8 \mathrm{~dB}$.

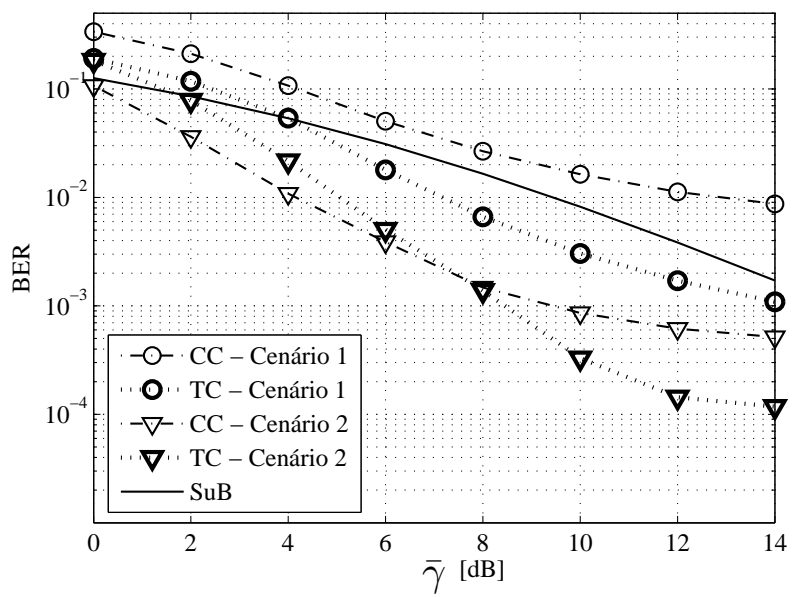

Fig. 6. Desempenho para decodificação Turbo $\left(N_{\text {iter }}=5\right)$ e Viterbi considerando carregamento do sistema 3 para cenários 1 e 2 .

\section{COMPlexidade COMPUTACIONAL}

A complexidade dos decodificadores depende diretamente do número de transições treliça por bit de informação. Para o codificador convolucional binário, existem apenas duas transições possíveis para cada um dos $2^{Q}$ estados. Com isso, pode-se aproximar a complexidade do decodificador de Viterbi por [16]:

$$
\mathcal{O}\{C C(n, Q)\}=n K 2^{Q}
$$

Considera-se que o número de transições treliça no algoritmo de decodificação Log-MAP é cerca de três vezes maior que a do algoritmo de Viterbi, pois percorre-se a treliça na direção direta e na direção reversa, além de realizar o cálculo logarítmico de saída suave. Para códigos Turbo é utilizado uma estratégia de decodificação que utiliza dois decodificadores (figura 2), com isso, a complexidade computacional da decodificação Turbo, utilizando-se do algoritmo Log-MAP pode ser aproximada por [16]:

$$
\mathcal{O}\{T C(n, Q)\}=6 N_{\text {iter }} n K 2^{Q}
$$

Para a análise do requisito de memória utilizada pelos decodificadores deve-se considerar o número de estados da treliça por bloco codificado. Para o codificador convolucional binário, observações realizadas para o algoritmo de Viterbi mostraram que todos os percursos sobreviventes dos estados correntes da treliça surgem, no máximo, dos $5 \times(Q+1)$ estados anteriores da treliça. Com isso, a cada instante da decodificação, apenas $5 \times(Q+1)$ transições são armazenadas na memória. Portanto, para o algoritmo de Viterbi o requisito de memória é dado por [16]:

$$
\operatorname{Mem}\{C C(n, Q)\}=5(Q+1) K 2^{Q}
$$

$\mathrm{O}$ algoritmo Log-MAP requer armazenar as variáveis $\gamma, \alpha$ e $\beta$. O requisito de memória para o algoritmo Log-MAP é também cerca de três vezes o requisito do algoritmo de Viterbi. Consequentemente, o requisito de memória da estratégia Turbo é de [16]:

$$
\operatorname{Mem}\{T C(n, Q)\}=3 I K 2^{Q}
$$

A tabela IV apresenta esses resultados considerando sistema S3 e que o decodificador Turbo apresenta uma menor complexidade em termos do número de transições na treliça por bit transmitido e também um menor requisito de memória quando comparado ao decodificador de Viterbi. Através das equações (12) e (13) e considerando o número de registradores $Q=8$ e 3 para os decodificadores de Viterbi e Turbo, respectivamente, obtém-se uma relação indicando que ao se utilizar um número de iterações $N_{\text {iter }} \leq 5$ para a estratégia Turbo, esta apresentará uma menor complexidade. Observase que o requisito de memória da estratégia Turbo diminui com o aumento da taxa de codificação, enquanto que para a decodificação convolucional, este requisito mantém-se constante, independentemente da taxa.

\section{TABELA IV}

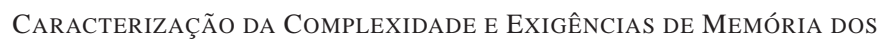
DiFERENTES DECODIFICADORES DE CANAL.

\begin{tabular}{|c|c|c|c|c|c|}
\hline $\begin{array}{c}\text { Código } \\
(n, Q)\end{array}$ & $\begin{array}{c}\text { Número } \\
\text { de estados } \\
\left(2^{Q}\right)\end{array}$ & $\begin{array}{c}\text { Algoritmo } \\
\text { Decodi- } \\
\text { ficação }\end{array}$ & $\begin{array}{c}\text { Tamanho } \\
\text { do Bloco } \\
(I)\end{array}$ & $\begin{array}{c}\text { Comple- } \\
\text { xidade } \\
(\mathcal{O})\end{array}$ & $\begin{array}{c}\text { Requisito } \\
\text { Memória } \\
(\mathrm{Mem})\end{array}$ \\
\hline \multicolumn{6}{|c|}{ Código Convolucional (CC - Convolutional Code) } \\
\hline $\mathrm{CC}(2,8)$ & 256 & VA & 280 & 5120 & 115200 \\
\hline $\mathrm{CC}(3,8)$ & 256 & VA & 184 & 7680 & 115200 \\
\hline $\mathrm{CC}(4,8)$ & 256 & VA & 128 & 10240 & 115200 \\
\hline \multicolumn{6}{|c|}{ Código Convolucional Turbo (TC - Turbo Convolutional Code) } \\
\hline TC $(2,3)$ & 8 & MAP & 282 & 4800 & 67680 \\
\hline TC $(3,3)$ & 8 & MAP & 186 & 7200 & 44640 \\
\hline TC $(4,3)$ & 8 & MAP & 130 & 9600 & 31200 \\
\hline
\end{tabular}

A figura 7 indica que o aumento da complexidade é linear com o aumento do número de usuários e taxa de codificação para ambos os algoritmos de detecção. Essa afirmação pode ser verificada através das equações (12) e (13). 


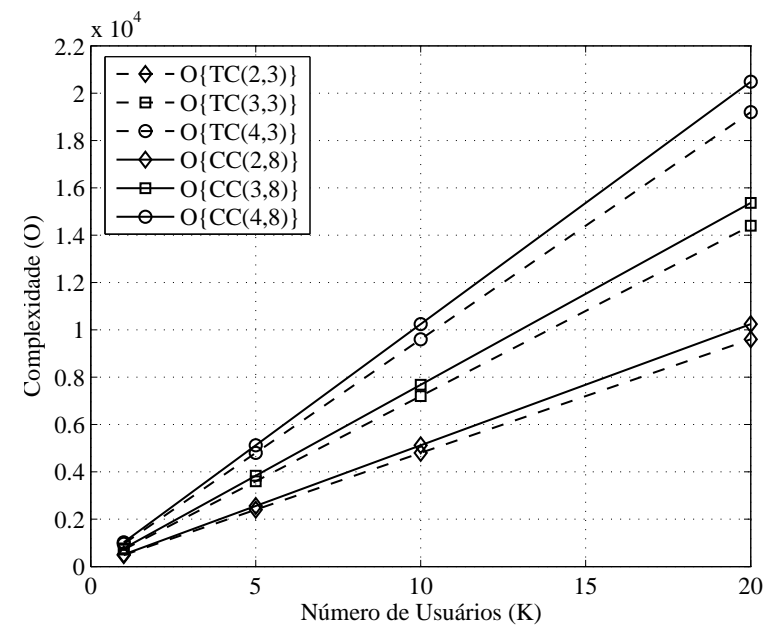

Fig. 7. Complexidade em termos do número de transições na treliça por bit de informação.

A figura 8 também indica que o aumento do requisito de memória é linear com o aumento do número de usuários e taxa de codificação para ambos os algoritmos de detecção. Além disso, observa-se que o requisito de memória decresce com o aumento da taxa de codificação para o decodificador Turbo.

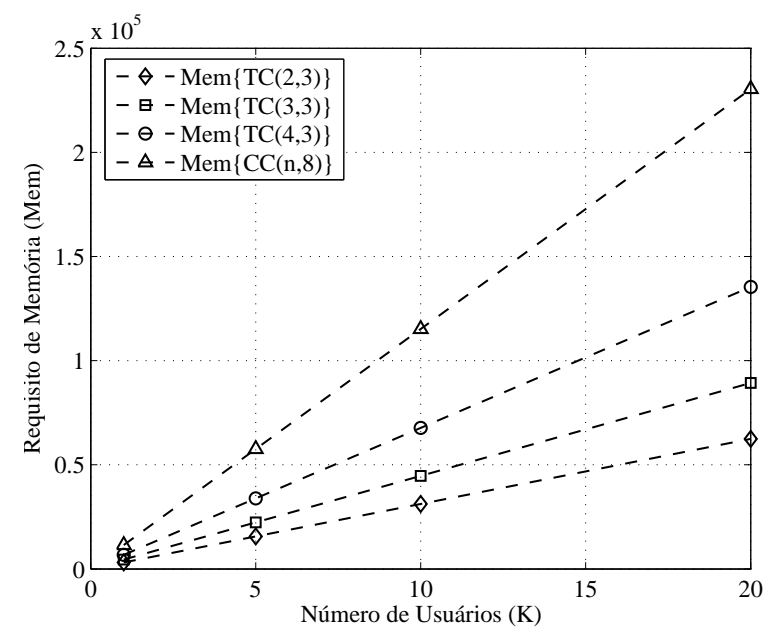

Fig. 8. Requisito de memória em termos do número de estados da treliça por bloco codificado.

Considerando os resultados apresentados na tabela IV e nas figuras 7 e 8 , percebe-se que a estratégia Turbo mostrase mais eficiente, pois apresenta uma menor complexidade e menor requisito de memória em relação ao decodificador convolucional para todos os sistemas analisados.

\section{CONCLUSÕES}

Neste trabalho propôs-se uma nova equação baseada no ajuste de curva do tipo sigmóide para a medida de confiabilidade do canal multipercurso com desvanecimento lento e seletivo em frequiência. Como resultado, obteve-se melhor consistência em termos de desempenho nas regiões de alta SNR em sistemas de múltiplo acesso DS/CDMA e detecção uniusuário $(\mathrm{SuD})$, operando sob diversas condições de carregamentos e codificação, mostrando ser uma escolha mais apropriada para a medida de confiabilidade do canal em comparação às equações sugeridas na literatura até o momento.

Comparou-se as estratégias de decodificação considerando como métricas o desempenho (BER), a complexidade e o requisito de memória. Resultados de desempenho MCS, de complexidade em termos de transições treliça por bit de informação e requisitos de memória mostraram que a decodificação Turbo é superior à convolucional (menor complexidade e menor requisito de memória), quando considerada a mesma taxa de codificação e apresenta melhor desempenho (BER) quando a quantidade de bits de informação é maior, desde que o número de iterações não seja superior a 5 e para um número de registradores específicos para a (de)codificação convolucional do padrão IS-95 e da (de)codificação turbo do padrão do cdma2000, respectivamente.

Assim, a estratégia de decodificação Turbo mostra-se mais atrativa pra a implementação na estação rádio-base de sistemas $3 \mathrm{G}$, pois apresentou um melhor compromisso desempenho e complexidade, quando comparado ao decodificador de Viterbi.

\section{REFERÊNCIAS}

[1] C. Berrou, A. Glavieux, P. Thitimajshima, "Near Shannon limit errorcorrecting coding and decoding", in Proc. ICC 1993, pp. 1064-1070.

[2] W. Koch, A. Baier "Optimum and Sub-Optimum Detection of Coded Data Disturbed by Time-Varying inter-Symbol Interference", IEEE Globecom, pp. 1679-1684, Dec. 1990.

[3] J. A. Erfanian, S. Pasupathy, G. Gulak, "Reduced Complexity Symbol detectors with Parallel Structures for ISI Channels", IEEE Trans. Communications, vol. 42, pp. 1661-1671, 1994.

[4] P. Robertson, E. Villebrun, P. Hoeher, "A Compararison of Optimal and sub-Optimal MAP Decoding Algorithms Operating in the Log Domain", Proc. Intern. Conf. Communications (ICC), pp. 1009-1013, Jun. 1995.

[5] J. Hagenauer, P. Hoeher, "A Viterbi algorithm with Soft-Decision Outputs and Its Applications", in Proc. of GLOBECOM'1989, Dallas, Texas, pp. 47.1.1-47.1.7, Nov. 1989.

[6] J. Hagenauer, "Source-controlled channel decoding",IEEE Transactions on Communications, vol. 43, pp. 2449-2457, September 1995.

[7] L. R. Bahl and J. Cocke and F. Jelinek and J. Raviv, "Optimal Decoding of Linear Codes for Minimizing Symbol Error Rate", IEEE Transactions on Information Theory, IT-20, pp. 284-287, March 1974.

[8] A. J. Viterbi, "Error Bounds for Convolutional Codes and an Asymptotically Optimum Decoding Algorithm", IEEE Transactions on Information Theory, vol. IT-13, pp. 260-269, April 1967.

[9] J. G. Proakis, M. Salehi, Communication Systems Engineering, PrenticeHall, 1994.

[10] J. Proakis. Digital Communications. 2. ed., McGraw-Hill, 1995.

[11] S. Verdú, Multiuser Detection. U.K.: Cambridge Univ. Press, 1998.

[12] S. Benedetto, D. Divsalar, G. Montorsi e F. Pollara, "Soft-Output Decoding Algorithms in Iterative Decoding of Turbo Codes", The Telecommunications and Data Acquisition Progress Report 42-124, Jet Propulsion Laboratory, Pasadena, California, pp. 63-87, Oct. 1995.

[13] S.Benedetto, D.Divsalar, G.Montorsi e F.Pollara, "A soft-input softoutput Maximum A Posteriori (MAP) module to decode parallel and serial concatenated codes", TDA progress report 42-127, Nov. 1996.

[14] J. P. Woodard, L. Hanzo, "Comparative Study of Turbo Decoding Techniques: An Overview", IEEE Transactions on Veicular Technology, Nov. 2000, Vol. 49, No. 6, pp 2208-2234.

[15] M. R. Soleymani, Y. Gao e U. Vilaipornsawai, Turbo Coding for Satellite and Wireless Communications, Kluwer Academic Publishers, 2002.

[16] T. H. Liew, L. Hanzo, "Space-Time Block Codes and Concatenated Channel Codes: A Historical Perspective and Comparative Study", Proc. of the IEEE, Febr. 2001.

[17] TIA/EIA/IS-2000-2, "Physical Layer Standard for cdma2000 Spread Spectrum Systems", Telecomunications Industry Association, 1999.

[18] 3rd Generation Partnership Project (3GPP), 3G TS 25.212, v3.5.0, Multiplexing and Channel Codinf (FDD). Dec. 2000. 